\title{
Interview with Tamim Asfour on "Anthropomatics: The State of the Art in Robotics and Challenges for Future Research"
}

\author{
Alexander Maedche
}

Published online: 4 February 2019

(c) Springer Fachmedien Wiesbaden GmbH, ein Teil von Springer Nature 2019

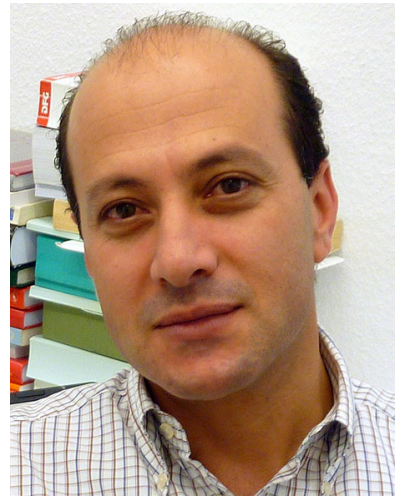

Prof. Dr. Tamim Asfour

Karlsruhe Institute of Technology

Institute for Anthropomatics and Robotics

High Performance Humanoid Technologies

Karlsruhe

Germany

asfour@kit edu

Tamim Asfour is full Professor of Humanoid Robotics at the Institute for Anthropomatics and Robotics, High Performance Humanoid Technologies at the Karlsruhe Institute of Technology (KIT). His research focuses on the engineering of high performance $24 / 7$ humanoid robotics

Prof. Dr. A. Maedche $(\bowtie)$

Karlsruhe Institute of Technology (KIT), Institute of Information Systems and Marketing (IISM), Karlsruhe Service Research Institute (KSRI), Karlsruhe, Germany

e-mail: alexander.maedche@kit.edu as well as on the mechano-informatics of humanoids. Tamim works on a synergetic integration of mechatronics, informatics and artificial intelligence methods into humanoid robot systems, which are able to predict, act and interact in the real world. In his research, he is reaching out and connecting to neighboring areas in large-scale national and European interdisciplinary projects in the area of robotics in combination with machine learning and computer vision. Tamim is the developer of the ARMAR humanoid robot family. He is scientific spokesperson of the KIT Center "Information - Systems - Technologies (KCIST)", president of the Executive Board of the German Robotics Society (DGR) and the Founding Editor-in-Chief of the IEEE-RAS Humanoids Conference Editorial Board.

BISE: Mr. Asfour, what are the key areas of your research? How has robotics research evolved and where do you see major breakthroughs in the past?

Asfour: My research focuses on engineering humanoid robots equipped with sensorimotor and cognitive abilities, which facilitate performing complex grasping and manipulation tasks, learning from human observation and sensorimotor experience, interacting and communicating with humans in a natural way as well as reasoning about consequences of actions. This requires the integration of mechatronics, control, computer vision, machine learning, and AI methods into complete cognitive architectures, which allow a bidirectional mapping between high-level symbolic knowledge and low-level sensorimotor control.

Robotics research has made huge progress in the last 50 years. Robotics have become a mature field of research and robotic technologies are rapidly transitioning from research labs to real world with unprecedented transformational effects on society. Robots are accurate, fast, and dexterous in structured environments and they perform a wide variety of tasks, with limited interaction with people 
and other robots, and where there is no need for a high level of autonomy, adaptability, learning and understanding. Progress has also been made in actuation, perception, grasping, and human-robot interaction resulting in robots that can be applied to a variety of different situations and applications ranging from manufacturing, logistics, search and rescue, healthcare, surgery and assistance.

Over the last years, robotics has changed dramatically from an engineering and control discipline into a transdisciplinary one that incorporates different areas to address challenges associated with building versatile robots with machine intelligence. It became clear that shortcomings of existing robot systems are due to the lack of rich physical interaction with the world and that design methodologies should go beyond task-orientation, exploiting interactions with the world and biological principles for dealing with the complexity of the world as well as principles of biologically inspired AI.

BISE: Can you shortly describe one of your currently running large-scale research projects?

Asfour: In the EU funded project SecondHands, we are developing collaborative humanoid robots which are able to perform maintenance tasks in warehouse environments in collaboration with human technicians. To this end, we developed the humanoid robot ARMAR-6 (Fig. 1), which provides a second pair of hands to assist maintenance technicians in their

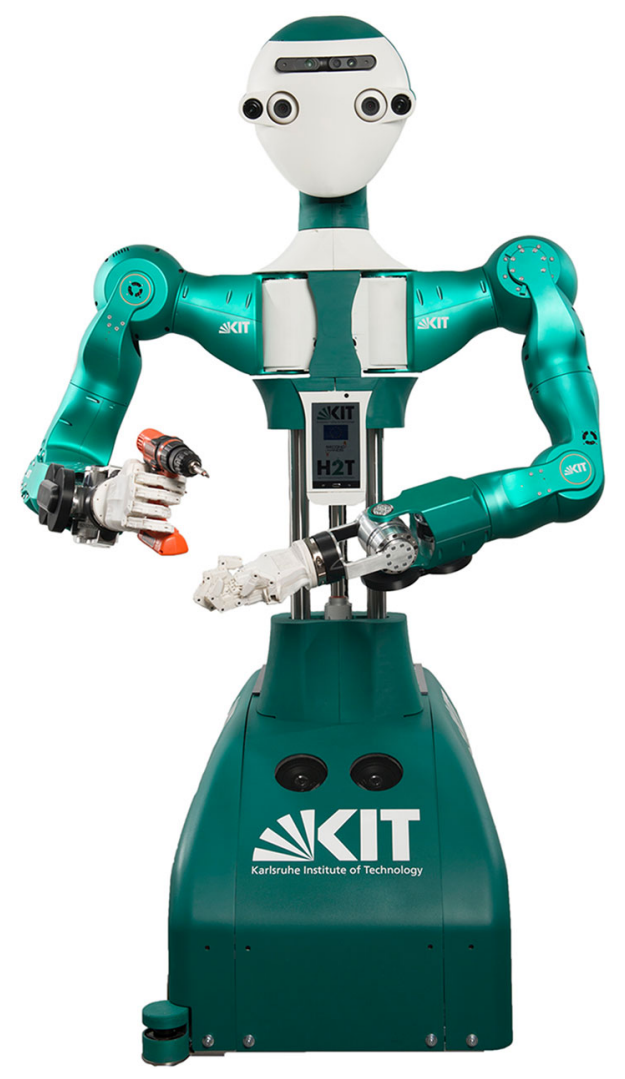

Fig. 1 Humanoid robot ARMAR-6 job. To this end, the robot has to recognize human activities, anticipate humans needs and pro-actively offer assistance when appropriate, in real-time, and in a dynamically changing real world. The application of such collaborative robots in automated warehouse environments will increase the efficiency and productivity of maintenance technicians in order to ensure a smooth running of production machinery thereby maximizing output and return on investment.

The project is funded by the European Union's Horizon 2020 Research and Innovation programme H2020 with partner from the Karlsruhe Institute of Technology (Germany), École Polytechnique Fédérale de Lausanne (Switzerland), Sapienza Università di Roma (Italy), University College London (United Kingdom) and Ocado Technology (UK).

BISE: The name of your institute at KIT is "Institute for Anthropomatics and Robotics"? Why do you emphasize "Anthropomatics" so explicitly in the name of your institute and what does it mean for you?

Asfour: The term » Anthropomatics "was coined as the science of symbiosis between human and machine and emphasizes the role of the human in developing artificial systems with advanced motor skills and cognitive abilities by integrating knowledge about humans with methods of computer science and artificial intelligence. By doing so we put the human in the center of our research towards intelligent machines being able to act and interact with humans in a natural way. We build and study humanoid robots to understand human intelligence and performance.

BISE: Some decades ago the robotics professor Masahiro Mori has coined the term "uncanny valley". It describes the non-linear relationship between the degree of human-like appearance of a robot and emotional responses of humans. What is your opinion about it and which type of research in robotics has be done in order to prevent "falling in the uncanny valley"?

Asfour: We are building humanoids, which are humanlike in terms of action and interaction abilities. We aim at versatile robots which (1) can perform a wide variety of tasks, (2) can act and interact in made-for-human environments and (3) can use made-for-human tools and objects. To the best of our knowledge, the human body is the best morphology, which can fulfil these tasks. Further, human-like motion allows humans to better predict robot's actions leading to fluent human-robot interaction. The question of the emotional responses towards robots that look or behave almost like a human is subject of many debates in humanoid robotics and human-robot interaction. It is an important question, which will play a central role in future mixed human-robot societies.

BISE: Where do you see important areas for interdisciplinary research with other disciplines and how could robotics benefit from it? 
Asfour: Robotics is an interdisciplinary research field. To create the next generation of robots and transfer scientific results into technology, research efforts from different research areas, such as engineering, computer science, life science, material science, and the humanities are needed. Understanding biological systems remains the source of inspiration for creating of robot systems able to solve complex tasks in a very efficient way, AI technologies can give rise to a new generation of robots with human-like or even superhuman performance, and novel materials and fabrication techniques would contribute to novel robot "bodyware". Social science and economics play an increasingly important role for providing social models for integrating robots into the society, ethical frameworks as socio-economic models of the application of robots.

BISE: The BISE community has a long tradition in the engineering of information systems from a social-technical perspective. Prominent examples are CSCW systems, ERP systems and BPM platforms, decision support systems and electronic markets. Where do you see promising synergies with robotics research? Are there any specific research challenges you could suggest?

Asfour: I am sure the robotics community can benefit from the work done in business and information system engineering to develop comprehensive methods, frameworks and tools for the support of robotics research and development as well as their successful deployment in organizations and the society. In think a deeper integration of robotics methods, frameworks and tools into the engineering and management lifecycle of (business) information systems is very relevant and interesting.

BISE: In the past, robots have demonstrated their automation potential in the industrial context. In contrast to industrial robots, service robots tightly cooperate with humans in order to co-create value. Looking at this from a socio-technical perspective, what are specific challenges you see from a research and practice point of view?

Asfour: From a technical perspective, it remains challenging to develop versatile humanoid robots with advanced sensorimotor and cognitive abilities which allow adaptation to novel situations and context. From a social perspective, we are still lacking (1) social models for introducing robots in the society of humans, (2) legal frameworks that regulate the wide application of robots in our environments and (3) ethics of human-robot interaction, of robot integration, of human-robot hybrids, of human augmentation.

BISE: We recently see a massive adoption of "software-based robots", e.g., in the form of text- and speechbased conversational agents. What can we learn from the field of robotics in the process of developing and implementing conversational agents in the real-world?

Asfour: Robots have to act and interact in a real complex and dynamically changing world. Thus, we have to take into account the complexity of the world arising from multimodal sensory information and uncertainties in perception and action execution. I think these are the topics where research on conversational agents can especially learn from robotics.

BISE: Looking at the future, how do you see the field of robotics evolving? What are the major challenges from your point of view?

Asfour: Robotics is a key technology of the twenty first century and will contribute to our understanding of natural intelligence and help to solve societal and economical challenges. Future robots will have to understand, communicate, and safely integrate into society in unprecedented ways. They will allow us to live longer active lives, improve productivity and reduce societal inequality, change education, and mitigate disasters. Future robots must be socially, ethically, energetically, and ecologically sustainable. The challenges range from novel materials and fabrications techniques for novel bodyware for safe, secure and trustworthy robotics, machine learning and AI methods tailored to real-world robot applications and socio-economical models for the successful integration of future robot generations into our society.

BISE: Mr. Asfour, thank you very much for your time and for this interview. 